\title{
High-Frequency Electronic Modeling Using Neural Networks
}

\author{
Qijun Zhang \\ School of Microelectronics, Tianjin University, Tianjin, China 300072
}

\begin{abstract}
We present an overview of neural network approaches for efficient modeling, simulation and optimization of high-frequency electronic and microwave circuits. Neural networks trained from electronic/microwave data are subsequently used for circuit simulation and optimization. A brief introduction of the fundamentals of neural network structures and neural model development issues including data generation, and neural network training are summarized. Applications of neural networks for modeling, simulation and optimization of transmission line networks for interconnect analysis on printed circuit board design are illustrated.
\end{abstract}

Keywords-modeling; neural networks; high-frequency electronics; microwave

\section{INTRODUCTION}

In the high-frequency/microwave electronics industry, the desire for manufacturibility-driven design and time-to-market demands powerful and efficient computer-aided design tools. With shrinking design margins and desire for first pass success, statistical analysis and yield optimization taking into account process variations and manufacturing tolerances in electronic devices and packages become necessary. This trend leads to massive as well as highly repetitive computational tasks during simulation, statistical design and optimization. However, combining large-scale circuit simulation and electromagnetic analysis inside an optimization and statistical design loop is extremely computationally challenging using conventional methods. The introduction of artificial neural networks to the high-frequency electronic and microwave design field [1-7] is an unconventional alternative to modeling, simulation and optimization problems in CAD. Neural networks has the speed of empirical models and the accuracy of detailed field solutions, offering both speed and accuracy in electronic design simulation and optimization. Neural models can be trained from data allowing model development even when component formulas are unavailable. Neural models are universal approximators allowing re-use of the same modeling technology for both linear and nonlinear problems.

In this paper, we present an overview of this area, and the trends in applying neural networks in microwave design. The neural network models are applied to modeling interconnects for printed circuit board design [8]. A brief introduction of the fundamentals of neural network structures and neural model development issues including data generation, and neural network training are summarized. Neural network models after training can be subsequently used for efficient simulation and optimization of high-frequency electronic and microwave circuits [9-10].

\section{NEURAL NETWORK MODELING}

We first describe the basic formulation of the modeling problem, and then express the problem in the neural network format. Let $\boldsymbol{x}$ represent a $N_{x}$-vector containing physical/geometrical parameters of an electronic device/circuit, e.g., gate length and gate width of a field effect transistor, or width and spacing of a transmission line. Let $\boldsymbol{y}$ represent a $N_{y}$-vector containing the responses of the device/circuit under consideration, e.g., drain current of a transistor, or mutual inductance of a transmission line. The physics/electromagnetic relationship between $\mathrm{x}$ and $\mathrm{y}$ can be represented as $\boldsymbol{y}=\boldsymbol{y}(\boldsymbol{x})$. This relation can be highly nonlinear and multi-dimensional. The theoretical model for this relationship may not be available (e.g., a new semiconductor device), or theory may be too complicated to implement, or the theoretical model may be computationally too intensive for online microwave design and repetitive optimization (e.g., 3D full-wave electromagnetic analysis inside a Monte Carlo statistical design loop). The purpose now is to develop a model in order to represent the $\mathrm{x}-\mathrm{y}$ relationship in a more efficient manner. To do this with neural networks, we teach/train a neural network to learn the microwave problem through a set of measured/simulated sample pairs called training data $\left\{\left(\boldsymbol{x}_{p}, \boldsymbol{d}_{p}\right), p \in T_{R}\right\}$, where $\boldsymbol{d}_{p}$ represents the measured/simulated output y for the input $\boldsymbol{x}_{p}$, and $\mathrm{T}_{\mathrm{R}}$ is the index set of training data. The neural network model is defined as [1].

$$
\tilde{\boldsymbol{y}}=\tilde{\boldsymbol{y}}(\boldsymbol{x}, \boldsymbol{w})
$$

where $\boldsymbol{w}$ contains $N_{w}$ trainable parameters of the neural network also called as the weight vector. For the purpose of training, we define error function $E(\boldsymbol{w})$ as

$$
E(\boldsymbol{w})=\sum_{p \in T_{R}}\left[e_{p}(\boldsymbol{w})\right]^{q}
$$

where q represents the qth norm and $e_{p}(w)$ is the error due to pth sample given by 


$$
e_{p}(\boldsymbol{w})=\left[\frac{1}{q} \sum_{k=1}^{N_{y}}\left|\tilde{y}_{k}\left(\boldsymbol{x}_{p}, \boldsymbol{w}\right)-d_{p k}\right|^{q}\right]^{\frac{1}{q}}
$$

where $d_{p k}$ is the kth element of dp and $\tilde{y}_{k}\left(\boldsymbol{x}_{p}, \boldsymbol{w}\right)$ is the kth output of the neural network for input sample $x_{p}$. The objective of neural network training is to find $w^{*}$ such that $E(\boldsymbol{w})$ is minimized. The definition of w, and the approach by

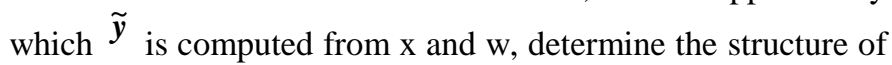
the neural network. The most popular form of neural network is the multi-layer perceptrons (MLP) structure. Other type of neural network structures can also be used for high-frequency electronic modeling applications, such as radial basis function networks [1].

\section{Training OF Microwave NeURAL Models}

In order to develop the neural networks models, we need to first identify the inputs and outputs of the modeling problem, so that the corresponding training data can be generated. For example, real and imaginary parts of S-parameters for linear circuit components, currents and charges for nonlinear component models, can be chosen as model outputs, and physical and geometrical parameters can be inputs of model. The next step is data generation. These data will be needed to teach the neural network and to test the accuracy of the model. In general, data generation means using a data generator to obtain the output dk, for each input sample xk. The total number of samples, $\mathrm{P}$, to be generated is chosen such that the developed neural model best represents the original problem. For microwave modeling applications, the data can be generated by measurement and/or simulation of the original problem. The sample distribution and the number of samples should be selected in such a way that the original problem $\boldsymbol{y}=\boldsymbol{f}(\boldsymbol{x})$ in the input space [xmin, xmax] is sufficiently represented by the data samples. The total number of data samples required depends on the nonlinearity of the problem, that is, highly nonlinear problems require more data. We also need to determine neural network structural issues such as number of layers, and number of neurons. For common circuit modeling problems, 3 or 4 layers are sufficient. For complicated and highly nonlinear modeling problems, more layers will be needed.

The concept of training is illustrated in Figure I. The purpose of neural network training is to adjust neural network internal weights such that the model outputs and the data are best matched.

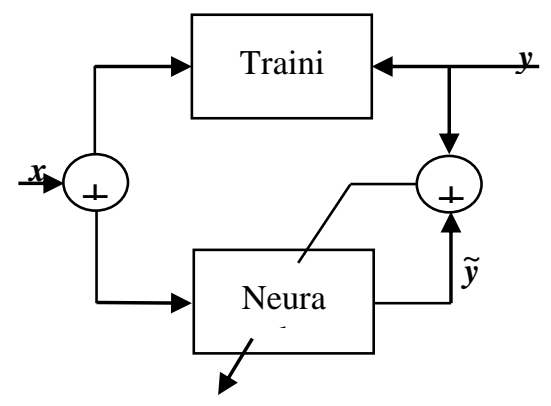

FIGURE I. ILLUSTRATION OF NEURAL NETWORK TRAINING. THE NEURAL NETWORK INTERNAL WEIGHTS ARE UPDATED USING THE INFORMATION OF ERROR BETWEEN MODEL AND TRAINING DATA BACK-PROPAGATED THROUGH THE NETWORK

For most of the microwave modeling problems, second order training algorithms such as conjugate-gradient, Quasi-Newton are usually more efficient than conventional backprogapation training. From optimization point of view, the training process adjusts $\boldsymbol{w}$ such that the error between neural model predictions and desired outputs is minimized.

After the training process is completed, we proceed to test the neural model with a separate set of data (from the same problem) which has not been used during training. If the error between neural model and test data is small, then the resulting model is ready to be used. If not, we need to train the neural network with different number of hidden neurons or adding more training data, until the final test error becomes small.

\section{EXAMPLE}

This examples shows neural network modeling for a transmission line network example. At high signal speeds, signal integrity becomes an important design criteria for the design of printed circuit board. The interconnections between electronic packagess in this case acts as multiconductor transmission lines, and the signals that travel through those interconnects can suffer from signal integrity problems such as delay, distortion, and crosstalk due to couplings between adjacent interconnects. The distributed network containing three 2-conductor transmission lines [8] will be modeled using neural networks. To solve the signal integrity problem of this circuit, the conventional method will involve eigen-value solutions of the transmission line matrices and mixed time/frequency domain analysis such as Fourier transform or numerical inversion of Laplace transform. The inputs to the neural model $\mathrm{x}$ contains six variables namely, the length and the separation between coupled conductors of each transmission line. The signal integrity responses y include the signal propagation delays of V1 and V2, and the magnitude of crosstalks Vc1 and Vc2. Figure II shows the neural network structure used in this example. 


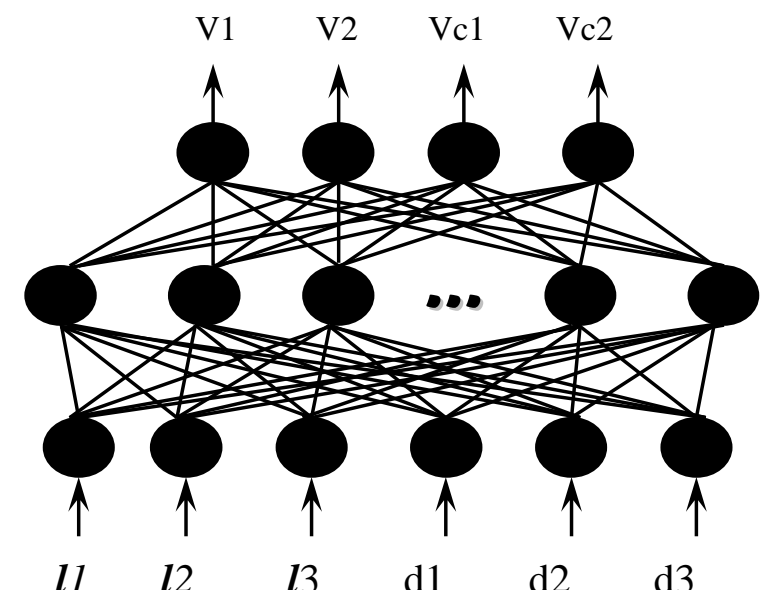

FIGURE II. AN EXAMPLE OF A NEURAL NETWORK MODEL FOR THE 2-CONDUCTOR TRANSMISSION LINE NETWORK EXAMPLE. LI AND DI ARE LENGTH AND SPACING BETWEEN COUPLED CONDUCTORS OF ITH TRANSMISSION LINE COMPONENT RESPECTIVELY. THIS NEURAL NETWORK WILL REPRESENT THE SIGNAL INTEGRITY BEHAVIOR WITH RESPECT TO DESIGN PARAMETERS IN THE TRANSMISSION LINE NETWORK

For this example, we use a circuit simultor to generate trainning data. A total of 500 training samples was generated. After training, the neural network can represent the behavior of the entire circuit, that is, for given length and spacing values, the neural model can predict delay and crosstalk. This model is very useful for signal integrity driven layout where the interconnect length and spacing are varied during optimization. This neural network can directly be used to simulate delay and crosstalk of such structure during printed circuit board design. In order to simulate the effect of manufacturing tolerances, we allow all the six input parameters to be statistical variables following Gaussian distribution with 5\% standard deviation. We then perform a Monte Carlo analysis with 500 interconnect circuits, each with randomly generated values of interconnect lengths and spacings. The design specifications for the four responses are $1.8 \mathrm{~ns}, 1.72 \mathrm{~ns}, 0.02 \mathrm{~V}$, and $0.02 \mathrm{~V}$ respectively. The specifications were severely violated before optimization and the yield was $0 \%$. In order to improve the yield, a yield optimization was performed using the neural network model. During optimization, the neural model is used to estimate the yield for a given circuit configuration, using 100 random circuit configurations distributed around the given configuration. The nominal values of the interconnect parameters (lengths and spacings) are then changed, effecting a change in all the 100 circuit configurations, and the yield is re-estimated. During this process, the weights of the neural network model are fixed, and the outputs of the neural model were forced to match or exceed the design specifications, by adjusting the neural model inputs. Neural network input-output evaluations are very fast, facilitating such a highly repetitive computation. The solution of yield optimization is the final nominal values of the neural model inputs (i.e., lengths and spacings of the transmission lines). After yield optimization, Monte Carlo analysis with 500 randomly generated circuit configurations are performed, and the final yield of $98 \%$ was achieved. A histogram of the delay is shown in Figure III. This figure is obtained by the neural network model developed. The 500 evaluations by the neural netwwork is much faster than performing 500 circuit simulations.

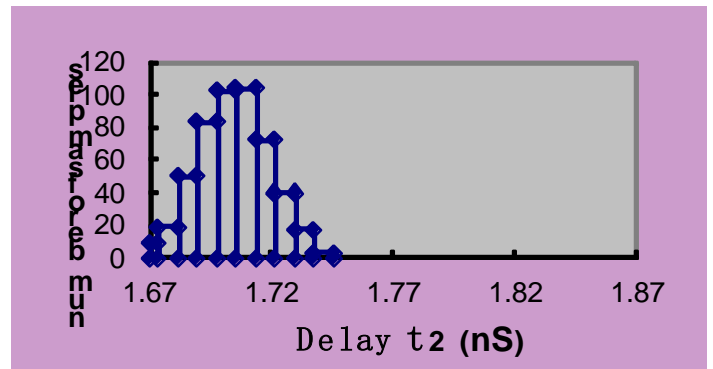

FIGURE III. HISTOGRAM OF SIGNAL DELAY FOR V2 FOR THE 2-CONDUCTOR TRANSMISSION LINE NETWORK EXAMPLE AFTER YIELD OPTIMIZATION USING NEURAL NETWORK MODELS. THERE ARE 500 TRANSMISSION LINE NETWORK CIRCUITS EACH WITH A RANDOMLY GENERATED SET OF PARAMETERS FOR THE TRANSMISSION LINE NETWWORK

\section{REFERENCES}

[1] Q.J. Zhang, K.C. Gupta and V.K. Devabhaktuni, "Artificial neural networks for RF and microwave design: from theory to practice," IEEE Transactions on Microwave Theory and Techniques, vol. 51, pp. 1339-1350, 2003.

[2] Q.J. Zhang and K.C. Gupta, Neural Networks for RF and Microwave Design, Artech House, Norwood, MA, 2000.

[3] W.C. Na and Q.J. Zhang, "Automated knowledge-based neural network modeling for microwave applications", IEEE Microwave and Wireless Components Letters, vol. 24, no. 7, pp. 499-501, July 2014.

[4] P.M. Watson, K.C. Gupta, and R.L. Mahajan, "Applications of knowledge-based artificial neural network modeling to microwave components", International Journal on RF and Microwave CAE, vol. 9, pp. 254-260, 1999.

[5] J. E. Rayas-Sanchez, "EM-based optimization of microwave circuits using artificial neural networks: The state-of-the-art," IEEE Transactions on Microwave Theory and Techniques, vol. 52, no. 1, pp. 420-435, Jan. 2004.

[6] M. Vai and S. Prasad, "Neural networks in microwave circuit design Beyond black box models," Int J. RF and Microwave CAE, vol. 9, pp. 187-197, 1999

[7] A. Patnaik, R.K. Mishra, G.K. Patra, and S.K. Dash, "An artificial neural network model for effective dielectric constant of microstripline," IEEE Transactionns on Antennas and Propagation, vol. 45, p. 1697, 1997.

[8] Q.J. Zhang, S. Lum and M.S. Nakhla, "Minimization of delay and crosstalk in high-speed VLSI interconnects," IEEE Transactions on Microwave Theory and Techniques, vol. 40, pp. 1555-1563, 1992.

[9] F. Feng, C. Zhang, J. Ma and Q.J. Zhang, "Parametric modeling of EM behavior of microwave components using combined neural networks and pole-residue based transfer functions," IEEE Transactions on Microwave Theory and Techniques, vol. 64, no. 1, pp. 60-77, Jan. 2016.

[10] W.Y. Liu, W.C. Na, L. Zhu, J.G. Ma and Q.J. Zhang, "Wiener-type dynamic neural network approach to the modeling of nonlinear microwave devices," IEEE Transactions on Microwave Theory and Techniques, vol. 65, no. 6, pp. 2043-2062, 2017. 